\title{
Influence of proceedings papers on citation impact in seven sub-fields of sustainable energy research 2005-2011
}

Ingwersen, Peter; Larsen, Birger; Garcia-Zorita, J. Carlos; Serrano-Lopez, Antonio Eleazar; Sanz-Casado, Elias

\section{Published in:}

Scientometrics

DOI:

10.1007/s11192-014-1335-2

Publication date:

2014

\section{Document version}

Early version, also known as pre-print

\section{Citation for published version (APA):}

Ingwersen, P., Larsen, B., Garcia-Zorita, J. C., Serrano-Lopez, A. E., \& Sanz-Casado, E. (2014). Influence of proceedings papers on citation impact in seven sub-fields of sustainable energy research 2005-2011. Scientometrics, 101(2), 1273-1292. https://doi.org/10.1007/s11192-014-1335-2 
Influence of proceedings papers on citation impact in seven sub-fields of sustainable energy research 2005-2011

\section{Peter Ingwersen, Birger Larsen, J. Carlos Garcia-Zorita, Antonio Eleazar Serrano- López \& Elias Sanz-Casado}

\section{Scientometrics}

An International Journal for all Quantitative Aspects of the Science of Science, Communication in Science and Science Policy

ISSN 0138-9130

Scientometrics

DOI 10.1007/s11192-014-1335-2

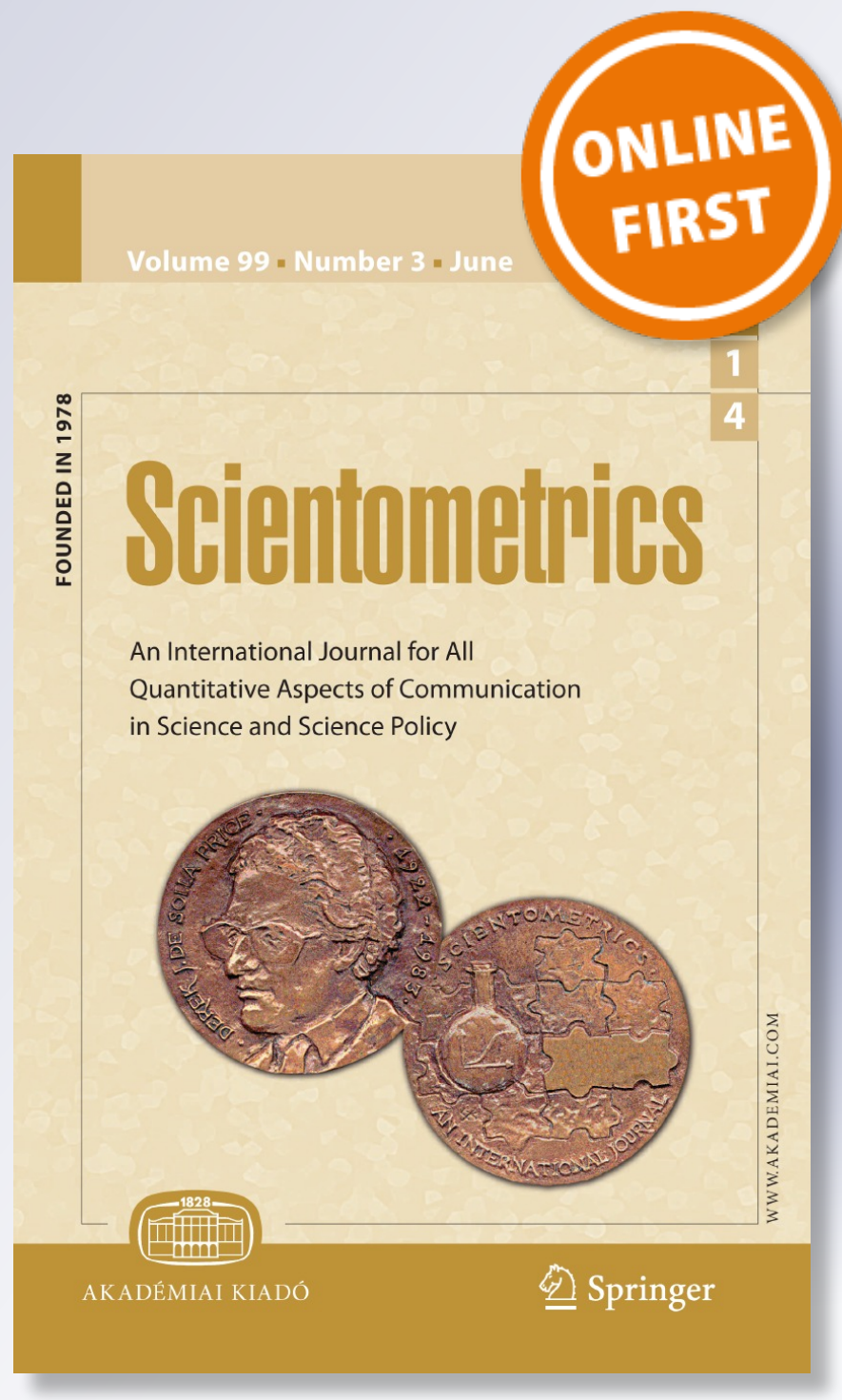

Springer 
Your article is protected by copyright and all rights are held exclusively by Akadémiai Kiadó, Budapest, Hungary. This e-offprint is for personal use only and shall not be selfarchived in electronic repositories. If you wish to self-archive your article, please use the accepted manuscript version for posting on your own website. You may further deposit the accepted manuscript version in any repository, provided it is only made publicly available 12 months after official publication or later and provided acknowledgement is given to the original source of publication and a link is inserted to the published article on Springer's website. The link must be accompanied by the following text: "The final publication is available at link.springer.com". 


\title{
Influence of proceedings papers on citation impact in seven sub-fields of sustainable energy research 2005-2011
}

\author{
Peter Ingwersen $\cdot$ Birger Larsen $\cdot \mathbf{J}$. Carlos Garcia-Zorita $\cdot$ \\ Antonio Eleazar Serrano-López • Elias Sanz-Casado
}

Received: 17 November 2013

(C) Akadémiai Kiadó, Budapest, Hungary 2014

\begin{abstract}
This paper analyses the following seven sub-fields of Sustainable Energy Research with respect to the influence of proceedings papers on citation patterns across citing and cited document types, overall sub-field and document type impacts and citedness: the Wind Power, Renewable Energy, Solar and Wave Energy, Geo-thermal, Bio-fuel and Bio-mass energy sub-fields. The analyses cover peer reviewed research and review articles as well as two kinds of proceeding papers from conferences published 2005-2009 in (a) book series or volumes and (b) special journal issues excluding meeting abstracts cited 2005-2011 through Web of Science. Central findings are: The distribution across document types of cited versus citing documents is highly asymmetric. Predominantly proceedings papers from both proceeding volumes as well as published in journals cite research articles (60-76\%). Largely, journal-based proceedings papers are cited rather than papers published in book series or volumes and have field impacts corresponding to research articles. With decreasing proceedings paper dominance in research fields the ratio of proceeding paper volumes over journal-based proceedings papers decreases significantly
\end{abstract}

P. Ingwersen $(\bowtie)$

Royal School of Information and Library Science, University of Copenhagen, Birketinget 6, 2300 Copenhagen S, Denmark

e-mail: clb798@iva.ku.dk

B. Larsen

Department of Communication, Aalborg University Copenhagen, A. C. Meyers Vænge 15, 2450 Copenhagen SV, Denmark

e-mail: birger@hum.aau.dk

J. Carlos Garcia-Zorita · A. E. Serrano-López · E. Sanz-Casado

Laboratory of Information Metric Studies (LEMI), Associated Unit IEDCYT-LEMI,

University Carlos III of Madrid, C/Madrid 126, Getafe, 28903 Madrid, Spain

e-mail: czorita@uc3m.es
A. E. Serrano-López
e-mail: aeserran@uc3m.es
E. Sanz-Casado
e-mail: elias@uc3m.es 
and the percentage of proceedings papers in journals citing journal-based proceedings papers over all publications citing journal-based proceedings papers decreases significantly (from $26.3 \%$ in Wind Power to $4 \%$ in Bio Fuel). Further, the segment of all kinds of proceedings papers (the combined proceedings paper types) citing all proceedings papers over all publications citing all kinds of proceedings papers decreases significantly (from $36.1 \%$ in Wind Power to $11.3 \%$ in Bio Fuel). Simultaneously the field citedness increases across the seven research fields. The distribution of citations from review articles shows that novel knowledge essentially derives directly from research articles (53-72\%) - to a much less extent from proceedings publications published in journals (9-13\%).

Keywords Document types - Proceedings papers - Research articles $\cdot$ Review articles · Citation impact · Citedness - Sustainable energy research · Renewable resources

\section{JEL Classification Q2}

\section{Introduction}

Commonly journal articles in the form of peer reviewed research articles and review articles are regarded the main vehicles for scientific communication in the natural science, bio-medical and some social science fields (Waltman et al. 2012). However, in several engineering fields as well as for computer science and other social science disciplines peer reviewed conference proceedings papers form the main scientific communication channel. With the inclusion of conference proceeding publications (CPCI-S and CPCI-SSH) in the Thomson-Reuters Web of Science citation index (WoS), and by application of the WoS Analytic Tools, it is possible to extract and observe how conference proceedings papers actually perform during a shorter time period compared to journal articles in selected research fields in a controlled manner.

The present analysis investigates seven fields of Sustainable Energy research published 2005-2009 with a citation window of max 7 years (2005-2011): the Wind Power and Renewable Energy subfields representing strong conference paper dependence (40-65\% of publications); Solar and Wave Energy subfields signifying medium conference dependence (26-39\%); and Geo-thermal, Bio-fuel and Bio-mass energy fields demonstrating low conference dependence $(<25 \%)$. The analysis distinguishes between two kinds of proceedings papers ${ }^{1}$ published in (a) (special) journal issues or (b) in book series/volumes, research articles and review articles, in total four document types. Other types containing editorials, book reviews, errata, meeting abstracts, etc. as defined in WoS are omitted, as is monographic material. As for journals WoS does not cover all conferences in the analysed Energy fields. However, according to (Thomson Reuters, ISI) the "most important and influential" conferences and conference proceeding volumes of the sustainable Energy fields, e.g. published in book series by ACM, IEEE and similar institutional sponsors, are covered and checked in a sample of records from the two proceedings paper groups extracted from WoS. The time slot analysed (2005-2009) corresponds to the period in which WoS through CPCI-S\&SSH has indexed its highest volume of proceedings papers

${ }^{1}$ In the remaining of the paper the notion 'proceeding papers' excludes the WoS document category 'Meeting abstracts'. 


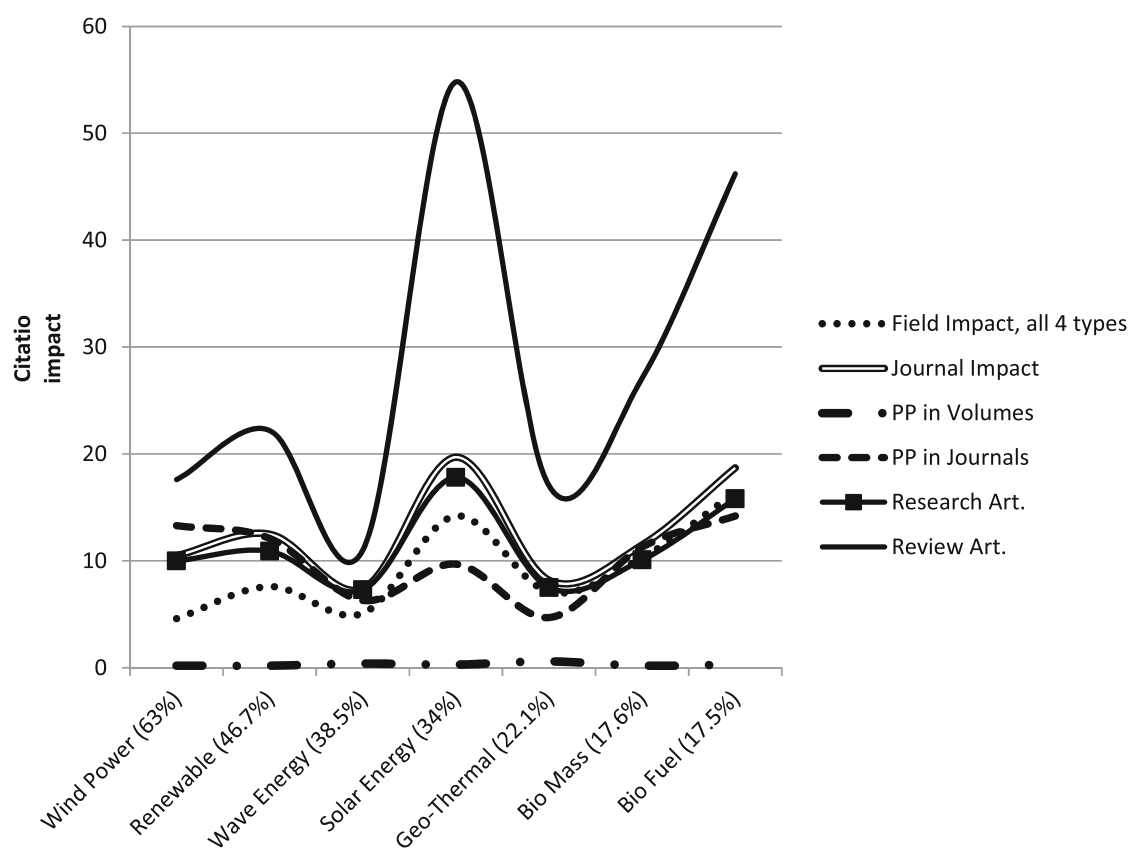

Fig. 1 Document type-related citation impact scores, seven Energy fields 2005-2009(11) (WoS, Jan. 2014)

with its peak at 479,000 papers in 2006 , according to analyses by Ingwersen and Larsen (2014, Fig. 1).

In an earlier study, the Research Evaluation and Policy Project (REPP) at the Australian National University (ANU), Bourke and Butler (1996) established a database covering all the publications from the Institute of Advanced Studies (IAS) and examined in detail citations in the journal literature accruing to all types of publications, including proceedings papers. The present study also observe the opposite citation flow, from proceedings papers to journal articles. Later studies of conference paper citation impact have demonstrated the feasibility of proceedings papers, e.g. Butler and Visser (2006) who investigated the degree to which WoS contributes adequate data with respect to a variety of document source types, including conference proceeding and meeting publications. Butler and McAllister (2009) examined metrics-based models for evaluating research in Chemistry and found that any metrics approach to performance evaluation has to use a discipline-specific suite of indicators. This proposal correlates to a very recent study by Mutz et al. (2013). They used a multi-level latent class analysis to define the kinds of research outputs in the shape of document types that can typically be expected from certain disciplines. Martins et al. (2010) tested comprehensive conference paper indicators in the Electrical Engineering and Computer Science fields, comparing to journal-based indicators. How proceeding paper citations are distributed across a range of document types in computer science was investigated by Wainer et al. (2011). They studied the references from all (predominantly proceeding) papers published in the ACM digital library 2006. They found that around $40 \%$ of the references were to earlier conference proceedings papers, around $30 \%$ were to journal papers, and around $8 \%$ were references to books.

Based on the latter findings founded on reference analyses one might form the hypothesis that in strong conference-dependent fields the proceedings papers themselves 
are the main contributor to the impact of the field or, at least, constitute a major supplier of citations to proceedings papers. In the present study this is measured by means of contingency tables and compared to citation impact and citedness scores across the four document types involved. Citedness signifies the portion of a given set of documents that have received at least one citation including self-citations.

One might also speculate that review articles in such conference-dominant disciplines would tend to cite conference papers, regardless publishing mode, rather than journal articles. However, a recent study of the conference-dominated engineering field Wind Power research 1995-2011 (Sanz-Casado et al. 2013) demonstrates that these hypotheses and ideas might not hold true for all conference-dependent fields. Hence the motivation for the present citation-based analyses, which aim at observing the characteristics of citations given to defined source documents of various types. Characteristics of the citing documents are, for instance, document type and which kinds of documents they cite, i.e., their citation pattern. An earlier study has presented some of these analyses (Ingwersen et al. 2013), which the present investigation extends in depth as well as range. If proceedings papers do play a crucial role in the knowledge distribution and crediting process they ought to be taken more into account, for instance in research evaluation studies.

The paper is organized as follows. The data collection and analysis methods are described, followed by the findings of the investigation. Initially we show the distribution of publications over the four document types across the seven selected Sustainable Energy research fields. This is followed by the distribution of citations, impact and citedness, including the distribution of citing documents, over the four document types in the seven fields. The findings are related to the degree of proceedings paper dependency for each of the seven fields as well as country and subject category characteristics. A discussion section and conclusions complete the article.

\section{Methodology}

The study made use of the already existing retrieval strategies and profiles developed and tested in the context of the SAPIENS project for the use in WoS. The SAPIENS Project (Scientometric Analyses of the Productivity and Impact of Eco-economy of Spain) has as main goal the analysis of scientific and technological capacities of Eco-economy in Spain 1995-2009, cited 1995-2011, seen in a global context through quantitative and qualitative R\&D indicators and is reported in Sanz-Casado et al. (2013).

The seven Energy research fields were extracted online in December 2012, October 2013 and January 2014 through WoS. Elaborated search profiles were executed. ${ }^{2}$ The following WoS citation databases were applied: SCI-EXPANDED, SSCI, CPCI-S, CPCISSH. For each field the online set of publications 2005-2009 was divided into the four document types examined in this study and analyzed by means of the WoS Analytic Tools for cited countries and WoS categories as well as citation distributions 2005-2011.

The distribution of document types across the seven selected fields is displayed on Table 1. A smaller share of documents (average $8 \%$ ) is indexed as proceedings paper as well as journal article within each field. A check demonstrated that they commonly were

\footnotetext{
${ }^{2}$ Example of search profile for wind power: TS $=$ ("wind power" OR "wind turbine*" OR "wind energy*" OR "wind farm*" OR "wind generation" OR "wind systems") AND PY $=(2005-2009)$. Refined by: document types $=$ (proceedings paper or article or review) and [excluding] Web of Science categories $=($ astronomy astrophysics $)$.
} 
proceedings papers published in thematic or special journal issues indexed by WoS. They are isolated as 'PP in journals' by means of refine and exclusion commands in WoS Analytic Tools. Thus they do not overlap with the research articles. Likewise proceedings papers published in book series or volumes are isolated as 'PP in volumes'. We checked if different time trends in publishing proceedings papers in the seven research fields occurred since that might bias the citation analyses. "Appendix A" section displays the annual distribution of the two proceedings paper types across the fields. It demonstrates a steady annual increase for "PP in volumes", with a very substantial output in 2009, and a more varied annual distribution of the smaller amount of "PP in journals" in all the seven fields. Thus, no citation bias exists between fields. The pattern for "PP in volumes" is contrary to the expected annual distribution pattern of CPCI-S and CPCI-SSH as observed in Ingwersen and Larsen (2014) and mentioned above.

Further, each sub-disciplinary set was sorted according to citation scores and the exact citedness ratio observed, Tables 2, 3, and 4. Intermediate analyses and calculations were necessary for each set of a document type to (1) exclude the 2012-2014 citations, (2) limit the citing set of publications to the required time period, and (3) define and logically isolate the distribution across the four document types of the citing set of publications. Thus no overlaps exist at document level in the contingency tables in which the four document types are exclusive. However, the exact number of citations from those types citing the original set cannot be secluded in Wos.

In case of sets too large for $\mathrm{WoS}$ to handle when generating online citation reports, i.e. sets above 10,000 items, the set was logically divided into subsets for which the analyses were aggregated later. The field of Solar Energy constitutes such a large set $(26,697$ documents). In total the analyses deal with almost 60,000 source documents (Table 1) and almost 700,000 citations. WoS Analytic Tools were also applied to extract the top-10 countries as well as the top-10 WoS categories published in the research articles and 'PP in volumes' per Energy field.

We have applied $\chi^{2}$ statistical tests and Pearson's Residuals to observe the significance of the trend results across the seven fields based on data from the contingency tables as well as the results from particular cells in each field analysis. Cramer's V is applied in order to measure the association strength using the "vcd" package implemented in $\mathrm{R}$ Software (Meyer et al. 2012).

\section{Findings}

Table 1 displays a 5-year snap shot of the seven sustainable Energy fields. It demonstrates the degree of dependency of both types of proceedings papers, which characterizes each field (percentages in bold). Evidently Wind Power research is mostly published through the proceedings paper channels (63\%); but also the Renewable Energy and Wave Energy fields are quite dependent on proceedings paper output (46.7 and $38.5 \%$ ). The engineering aspects of those fields are probably the reason for this dependency-see example Table 7. The remaining Energy fields under analysis are more science-like in their publication profiles being increasingly research article dominant. The annual distribution of proceedings papers published in the seven research fields are given in "Appendix A" section.

A closer look at Table 1 reveals a particular trend: The ratio of 'PP in volumes' over 'PP in journals' decreases with decreasing proceedings paper dominance in the research fields. From 13.7 in Wind Power (58.7/4.3) over 5.8 in Renewable Energy, 2.0 in Solar Energy to 1.6 in Bio Mass research. 







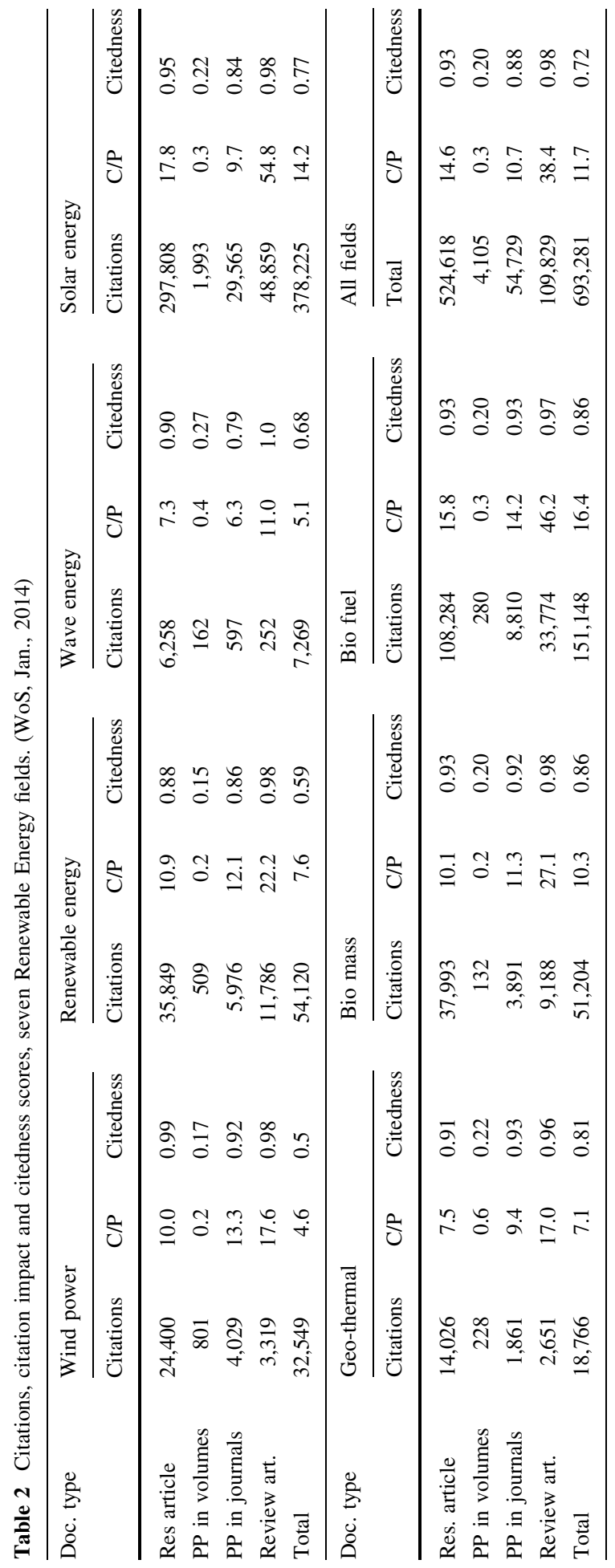




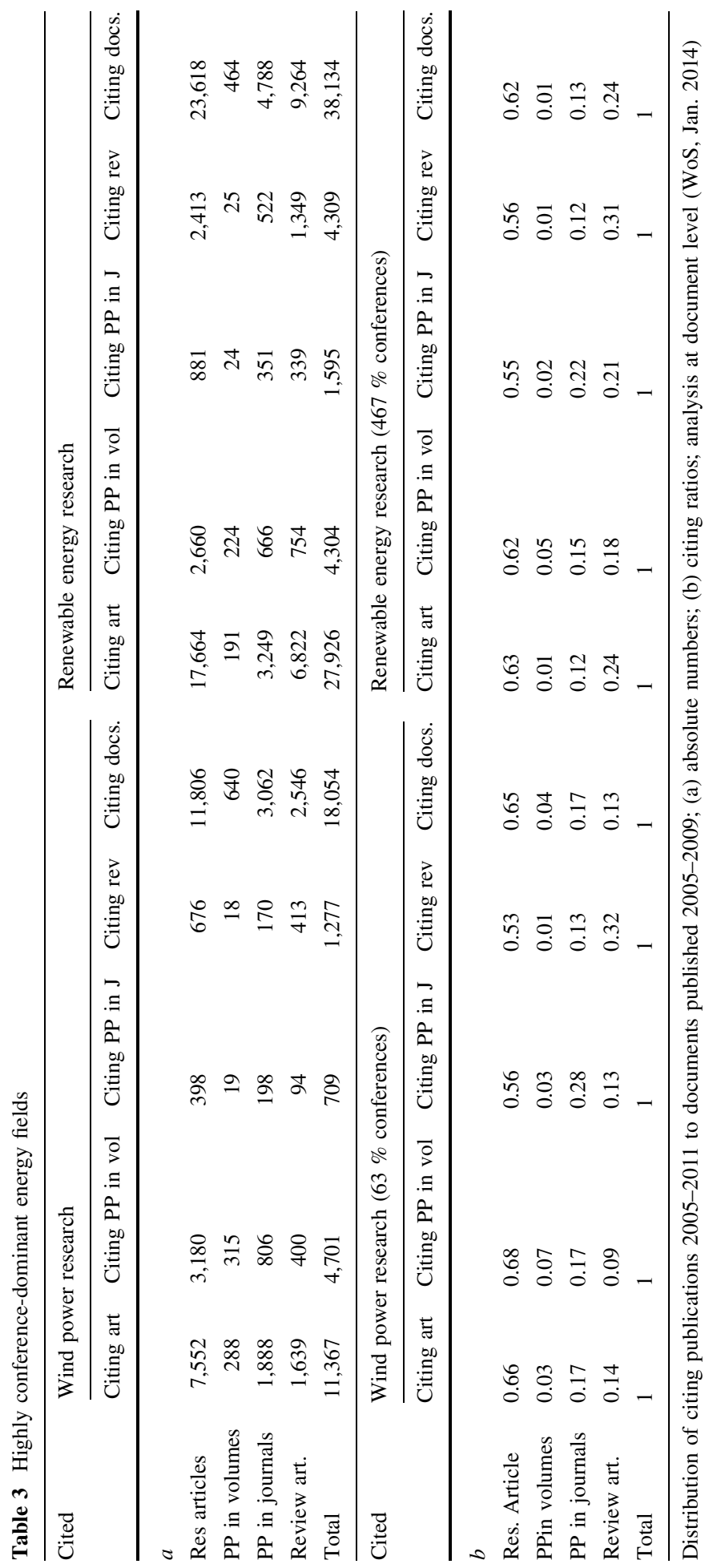




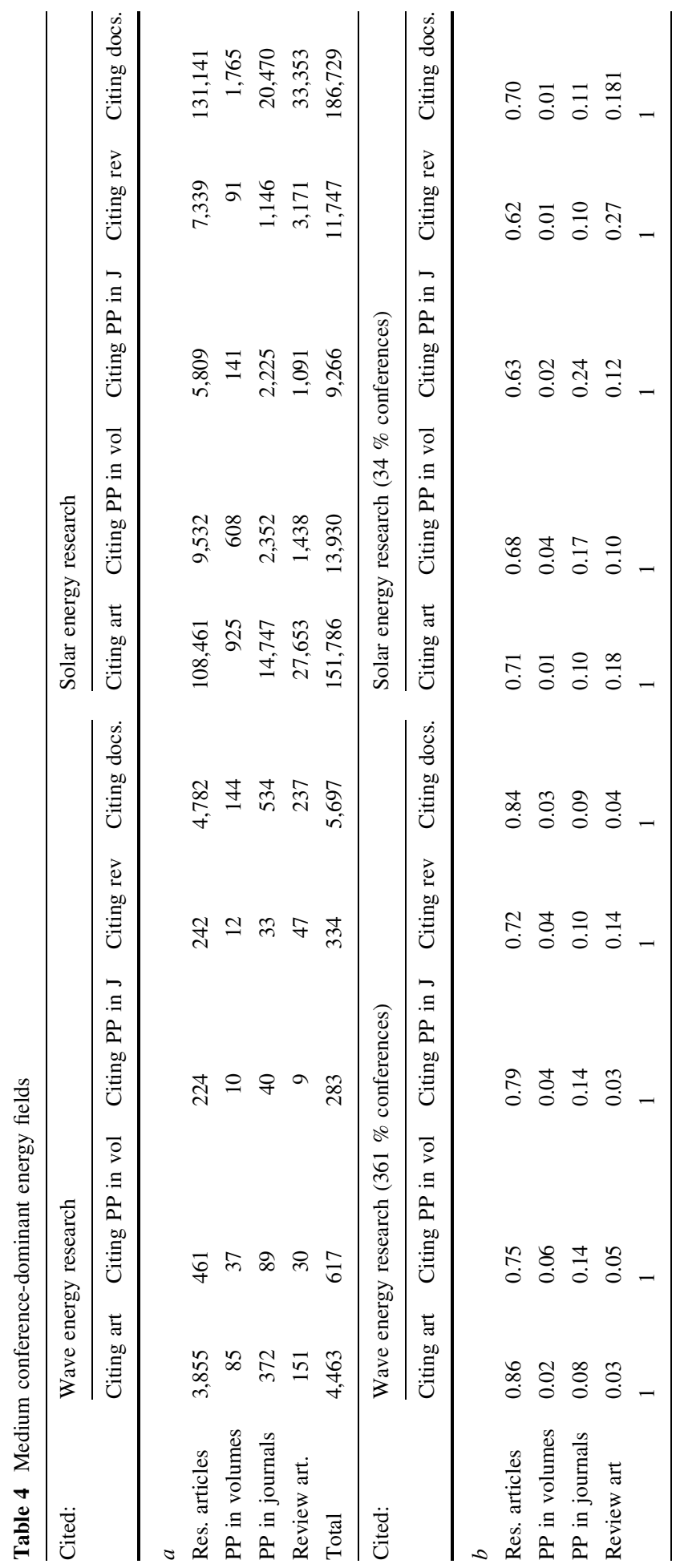




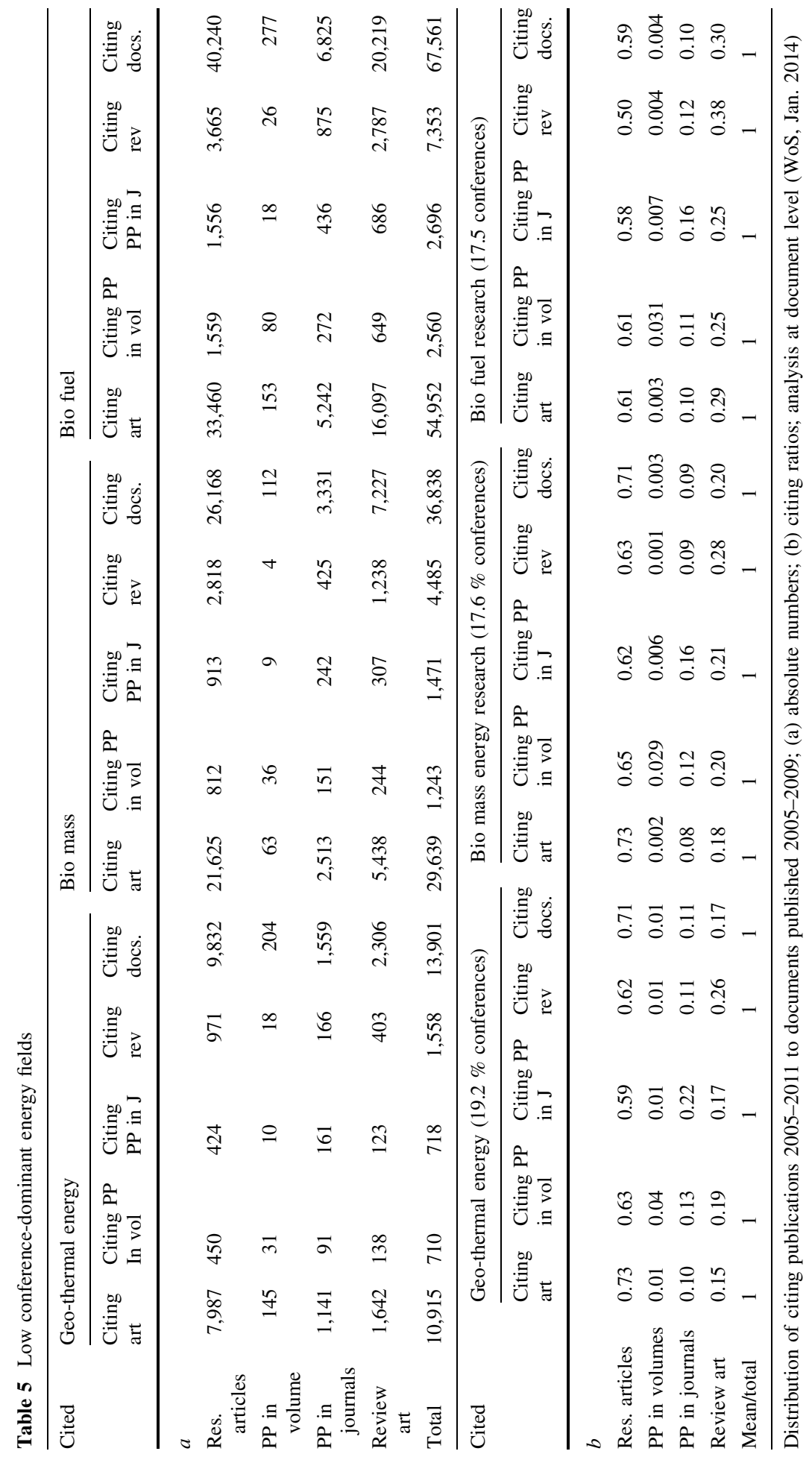


Table 6 Values of $\chi^{2}$ test statistics and Cramer's V for the seven renewable energy disciplines

\begin{tabular}{ll}
\hline Wind power research & $\chi^{2}(9, \mathrm{~N}=18,054)=695.84, p<.01$, Cramer'sV $=0,113$ \\
Renewable energy research & $\chi^{2}(9, \mathrm{~N}=38,134)=1,024.03, p<.01$, Cramer'sV $=0,095$ \\
Wave energy research & $\chi^{2}(9, \mathrm{~N}=5,697)=169.89, p<.01$, Cramer's $\mathrm{V}=0,10$ \\
Solar energy & $\chi^{2}(9, \mathrm{~N}=186,729)=5,465.7, p<.01$, Cramer'sV $=0,099$ \\
Geo-thermal energy & $\chi^{2}(9, \mathrm{~N}=13,901)=275.49, p<.01$, Cramer'sV $=0.081$ \\
Bio mass & $\chi^{2}(9, \mathrm{~N}=36,838)=659.83, p<.01$, Cramer'sV $=0.077$ \\
Bio fuel & $\chi^{2}(9, \mathrm{~N}=67,561)=963.03, p<.01$, Cramer'sV $=0.069$ \\
\hline
\end{tabular}

$\mathrm{CV}=21.666$

Table 7 Country profiles for Wind Power and Wave Energy publications 2005-2009

\begin{tabular}{|c|c|c|c|c|c|c|c|c|c|c|c|}
\hline \multicolumn{6}{|c|}{ Wind power field 2005-2009 } & \multicolumn{6}{|c|}{ Wave energy field 2005-2009 } \\
\hline \multirow{2}{*}{$\begin{array}{l}\text { Res. } \\
\text { articles } \\
\text { Country }\end{array}$} & \multicolumn{2}{|c|}{2,449} & \multirow{2}{*}{$\begin{array}{l}\text { PP in } \\
\text { volumes } \\
\text { Country }\end{array}$} & \multicolumn{2}{|c|}{4,181} & \multirow{2}{*}{$\begin{array}{l}\text { Res. } \\
\text { articles } \\
\text { Country }\end{array}$} & \multicolumn{2}{|l|}{861} & \multirow{2}{*}{$\begin{array}{l}\text { PP in } \\
\text { volumes } \\
\text { Country }\end{array}$} & \multicolumn{2}{|l|}{554} \\
\hline & no. & $(\%)$ & & no. & $(\%)$ & & no. & $(\%)$ & & no. & $(\%)$ \\
\hline USA & 396 & 16.2 & $P R$ China & 885 & 21.2 & USA & 280 & 32.5 & USA & 80 & 17.4 \\
\hline England & 196 & 8.0 & USA & 508 & 12.2 & England & 83 & 9.6 & Japan & 32 & 7.0 \\
\hline Denmark & 189 & 7.7 & Japan & 269 & 6.4 & PR China & 62 & 7.2 & PR China & 31 & 6.8 \\
\hline Canada & 171 & 6.9 & Germany & 242 & 5.8 & Australia & 59 & 6.9 & England & 29 & 6.3 \\
\hline Germany & 165 & 6.7 & Canada & 194 & 4.6 & Japan & 50 & 5.8 & Portugal & 29 & 6.3 \\
\hline Spain & 165 & 6.7 & Denmark & 129 & 3.1 & France & 47 & 5.5 & South Korea & 24 & 5.2 \\
\hline Japan & 128 & 5.2 & Spain & 129 & 3.1 & Canada & 46 & 5.3 & Canada & 21 & 4.6 \\
\hline PR China & 122 & 5.0 & India & 127 & 3.0 & Germany & 39 & 4.5 & Scotland & 18 & 3.9 \\
\hline Turkey & 111 & 4.5 & England & 119 & 2.8 & India & 34 & 3.9 & Australia & 15 & 3.3 \\
\hline Scotland & 87 & 3.6 & France & 102 & 2.4 & Italy & 29 & 3.4 & Italy & 14 & 3.1 \\
\hline
\end{tabular}

Prominent variance in bold + italics (WoS, jan., 2014)

Citation impact patterns and proceedings paper field dominance

Citation impact is calculated by dividing the number of citations, Table 2, by number of publications, Table 1 . Citedness as defined above is the ratios directly observed in WoS. According to Table 2 the citation impact varies substantially from document type to document type and across the seven sustainable Energy fields. However, in all the seven fields the 'PP in volumes' citation impact and citedness scores are extremely low (0.2-0.6; $0.15-0.27$ ) although $2 / 3$ of this type of proceedings papers have had four or more years to obtain citations in all the fields, "Appendix A" section.

Figure 1 summarizes the development of the citation impact scores for each discipline and each document type as well as a combined research and review article impact score, named 'Journal Impact'. The 'Journal Impact' equals the diachronic citation impact of a given field when the proceedings papers are omitted from a research evaluation analysis. The seven fields are sorted according to decreasing proceedings paper dominance.

Figure 1 indicates the importance of inclusion of the proceedings papers in research evaluation analyses. Whilst the overall field impact, the 'Journal Impact' and the research article impact scores for the two article-dominant Energy fields (Bio-mass; and Bio-fuel) are quite close in values, the five other Energy fields demonstrate an often wide gap 
between field impact and 'Journal Impact' scores. Although this trend is not statistically significant the observations suggest that in the proceedings paper dominant fields of Wind Power and Renewable Energy, as well as in the Wave Energy sub-field, the gap may occur owing to the negative influence of the numerous but low-cited 'PP in volumes' publications. In two of these fields the impact of 'PP in journals' is even higher than that of the research articles and thus contribute positively to the field impact.

In Solar Energy (medium proceedings paper dependent) and Geo-thermal energy (low proceedings-dependency) both types of proceedings papers influence negatively on the field impact-Table 2; Fig. 1. If 'PP in journals' and 'PP in volumes' are omitted from a citation-based evaluation of the Energy research fields the resulting impact scores are fairly accurate in the two article-dominant fields but, for different reasons, substantially misleading in the latter five fields.

Further, Table 2 demonstrates that the citedness values of 'PP in journals' constantly are very high compared to the citedness of the 'PP in volumes' type. Figure 2 depicts the citedness scores for each field as well as for the two proceedings paper types and research articles. The general trend is a continuous significant increase of field citedness with increasing article dependency, from $50 \%$ in Wind Power to $86 \%$ in Bio Fuel, $\chi^{2}$ (6, $\left.\mathrm{N}=507)=16.208, p<.05 ; \mathrm{CV}^{3}=12.592 ; \mathrm{R}^{2}=0.97\right)$. The citedness scores for the research articles and the two proceedings paper types demonstrate some variation but no particular patterns.

\section{Citations to and from document types}

Tables 3, 4, and 5 demonstrate the distribution of citations from the pool of citing publications to each of the four different types of source (cited) documents across the seven fields, displayed in descending order of conference dominance.

The central (vertical) trend is that in all the analysed sustainable Energy fields both types of proceedings papers essentially cite research articles. Between 55 and $84 \%$ of all the citing publications cite research articles. The 'PP in journals' type only cites 'PP in journals' at a smaller scale across the seven fields (11-28 \%) and virtually no citations go to 'PP in volumes' papers. The 'PP in volumes' type itself only scarcely cites 'PP in journals' (11-17\%). With respect to degree of proceedings paper dominance only one weak vertical pattern is observable: with decreasing proceedings paper dominance the percentage of 'PP in volumes' citing both types of proceedings papers decreases gradually from $21 \%$ (Wind Power) to $14.1 \%$ (Bio Fuel).

The Tables $3 b, 4 b$, and $5 b$ inform that only between $10 \%$ (in most fields) and $13 \%$ (Wind Power) of the citing review articles target 'PP in journals'; almost none goes to the 'PP in volumes' type. Most of the citations given by review articles extent to research articles, which thus can be regarded as the most significant source of novel knowledge in the Renewable Energy fields. Overall, we can see from the $\chi^{2}$ test statistics and their respective values of Cramer's $\mathrm{V}$ of tables $3 \mathrm{a}, 4 \mathrm{~b}$, and $5 \mathrm{a}$ that no or very weak associations exist between the type of document citing and document cited.

However, from the values of Pearson's residuals [(observed - expected)/sqrt (expected)] of contingency Tables 3a, 4b, and 5a, "Appendix B" section, one can observe some significant associations between certain cited and citing documents. In this way, one discerns that in all analysed areas there are positive associations in the observed values for "PP in volumes" as cited and citing documents and for "PP in journals" citing "PP in

\footnotetext{
${ }^{3} \mathrm{CV}$ signifies the critical value at a given $p$ value and degree of freedom.
} 


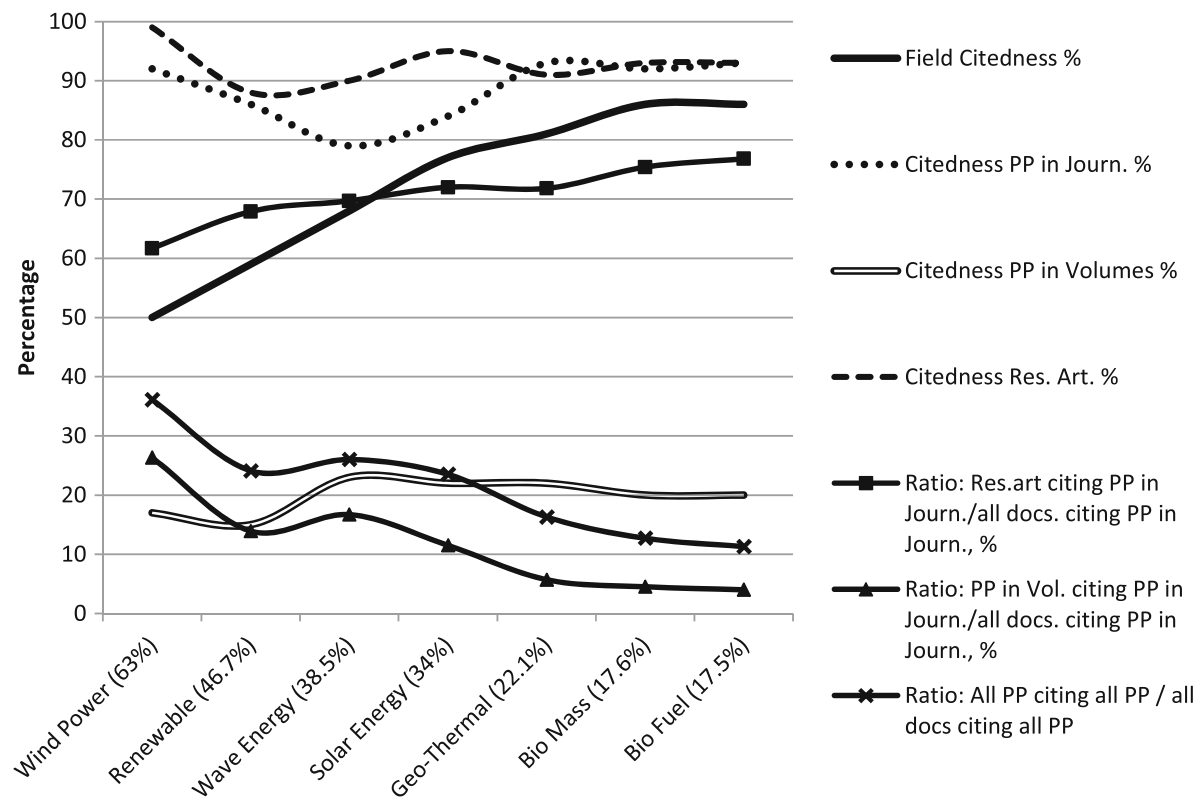

Fig. 2 Citedness per research field for document types and selected ratios of document types citing 'PP in journals' (WoS, Jan., 2014)

journals". The largest association occurs in Solar Energy between "PP in volumes" as cited and citing documents (Pearson's residual $=41.5$ ). However, the values do not correspond to the degree of proceedings paper dominance. Thus, the hypothesis that in research areas of strong proceedings paper dependency more citations than expected by chance are received by proceedings papers from proceedings papers than in less proceedings paper dependent areas does not hold. Only the mentioned more general significant positive association for the two proceedings paper types holds across all areas, independently from the degree of proceedings paper dominance.

Moreover one observes the existence of a negative association in all disciplines of research articles citing "PP in volumes" and "PP in journals", again Solar Energy being the area in which the observed values are well below the expected ones by chance (Pearson residual $=-13.7$ and -14.7 ).

One may in addition use the data Tables $3 b, 4 b$ and $5 b$ to calculate the share of citations given to 'PP in journals' from the different document types (i.e., calculating the horizontal ratios, not shown on tables). Figure 2 displays two fairly strong significant trends with respect to decreasing proceedings paper dominance of fields-and thus increasing article dependency: (1) the segment of all proceedings papers citing all proceedings papers over all publications citing proceedings papers (i.e. the combined 'PP in volumes' and 'PP in journals') decreases slowly but significantly (from $36.1 \%$ in Wind Power $(315+19+806+198 /(3,062+640))$ to $11.3 \%$ in Bio Fuel $) ;(2)$ the percentage of 'PP in volumes' citing 'PP in journals' over all publications citing 'PP in journals' decreases significantly (from $26.3 \%$ (Wind Power: 806/3,062) to $4 \%$ (Bio Fuel)). Figure 3 demonstrates the two correlations in scatter plot. Both display high $\mathrm{R}^{2}$ scores. 

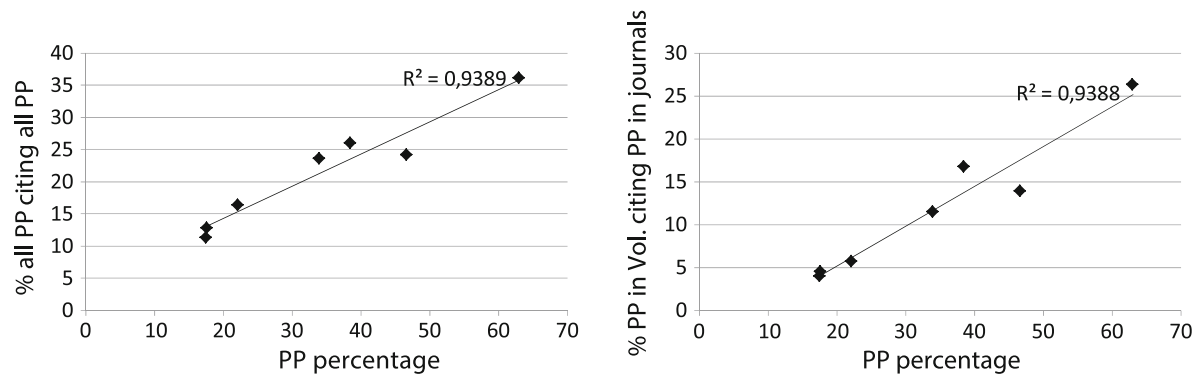

Fig. 3 The correlation between the percentage of all PP citing all PP over all publications citing all PP (left); and the percentage of 'PP in volumes' citing 'PP in journals' over all publications citing 'PP in journals' (right)

A third trend displaying a similar but non-significant pattern concerns the increase in the percentage of research articles citing 'PP in journals' over all publications citing 'PP in journals', from $61.7 \%$ in Wind Power $(1,888 / 3,062)$ to $76.8 \%$ in Bio Fuel. The testing shows that the trend might be due to random variation.

Country and subject matter characteristics of Energy publication types

The idea behind the analyses of top-10 publishing countries and WoS categories for research articles versus the 'PP in volumes' type is to observe possible profile discrepancies between the two prevailing document types. As long as the pair of country (or topical) profiles derived from research articles and 'PP in volumes' are very similar for a field, the two document types behave correspondingly with respect to a) production and b) top research foci. If, notwithstanding, discrepancies are noticeable they may signify reasons for certain citation phenomena or other anomalies observed.

Except for Wind Power and Wave Energy, Table 7, the country profiles in all the remaining five sustainable Energy fields demonstrate quite similar arrays as well as productivity shares for the two document types. In those five fields research articles and 'PP in volumes' contribute proportionally to the overall field citation impact, i.e., the same countries publishing research articles obtain simply far less citations to their 'PP in volumes' type.

In Wind Power P R China constitutes the outlier in the publication profile 2005-2009 by producing $21 \%$ of the world 'PP in volumes' publications (as indexed by WoS). China only publishes $5 \%$ of the research articles in the field during the same analysis period. For the 'PP in volumes' category produced in China and indexed by WoS the citation impact is almost zero (0.09). This is a main reason why the field impact for 'PP in volumes' type in Wind Power is very low (Table 2: 0.17) and the overall field impact is correspondingly deflated (4.6). The Wind Power citation impacts for the top-7 countries in the 'PP in volumes' type are compared to the corresponding impact scores for research articles and 'PP in journals', Table 8 . In contrast to the 'PP in volumes' type the Chinese impact in the other document types are more significant but still insubstantial compared to the other topcountries' 'PP in journals' scores, which constantly supersede the corresponding research article impact values.

In Wave Energy research the US 'PP in volumes' production is less dominant than research articles. In this field Portugal and Scotland are important 'PP in volumes' 
Table 8 Wind Power impact scores for top-7 countries, sorted by number of publ. in 'PP in volumes' column, Table 6 (WoS, Jan., 2014) in bold + italics

\begin{tabular}{llcc}
\hline Wind power & PP in volumes & PP in journals & Research articles \\
\hline P R China & $\mathbf{0 . 0 9}$ & $\mathbf{7 . 0 4}$ & 10.23 \\
USA & 0.20 & 15.13 & 12.52 \\
Japan & 0.13 & 16.09 & $\mathbf{7 . 1 1}$ \\
Germany & 0.45 & 9.52 & 8.85 \\
Canada & 0.30 & 15.91 & 10.90 \\
Spain & 0.24 & 24.00 & $\mathbf{1 4 . 0 9}$ \\
Denmark & $\mathbf{0 . 4 7}$ & $\mathbf{3 0 . 7 7}$ & 13.04 \\
\hline
\end{tabular}

producers, compared to their position outside top-10 as research article providers. As in Wind Power this Energy field displays a pattern of far less impact for the 'PP in volumes' type compared to research articles and the 'PP in journals' type.

Table 9 displays the prominent variances with respect to the pairs of topical profiles using WoS Subject Categories. The most significant differences are found in the Wave and Solar Energy fields. Wind Power and, to an extent, the Bio Mass Energy areas demonstrate minor discrepancies with respect to the ranking and kind of categories. In the remaining three Energy fields (Renewable Energy, Geo-Thermal and Bio Fuel) research articles and the 'PP in volumes' type demonstrate very similar profiles implying that the same categories contribute proportionally to the overall field citation impact scores.

The observed profile differences in the two Energy fields, Table 9, are mainly constituted by a stronger connection to and higher weights of the engineering categories in the 'PP in volumes' array of topics compared to that of research articles. The latter displays more citation-rich science-related subject areas. This is evident in the Wave Energy field with Oceanography vs. Engineering Ocean as top-categories and in Solar Energy by Engineering Electrical and Electronic and other engineering categories in 'PP in volumes', Table 9. In Wind Power (not shown) the area Engineering Electrical and Electronic is ranked second in the research articles (27.2\% world shares) but as top-category in the array of 'PP in volumes' $(64.6 \%)$. In addition, 'PP in volumes' in Wind Power deals uniquely and heavily with Automation and Control Systems and several low-cited Computer Science sub-categories. In the Bio Mass field (not shown) the Environmental and Mechanical Engineering fields are ranked 3-4 among the 'PP in volumes' publications but do not form part of the top-10 categories in research articles.

\section{Discussion}

The presented findings concern the Core Web of Science citation index. ${ }^{4}$ In other citation index configurations the resulting trends and patterns might thus differ slightly. According to analyses of CPCI-S and CPCI-SSH (Ingwersen and Larsen 2014, Fig. 1) the amount of both types of proceedings papers in the indexes peaked 2006 followed by a steady decline. The present analysis covers a snap shot of 2005-2009 publications surrounding the peak. However, "Appendix A" demonstrates that a large proportion of "PP in volumes" publications actually are published in 2008-2009, not in 2006 across all the seven fields. This fact has influenced the findings considering impact and citedness. They are quite low

\footnotetext{
${ }^{4}$ Excluding the recent addition of book citation indexing.
} 


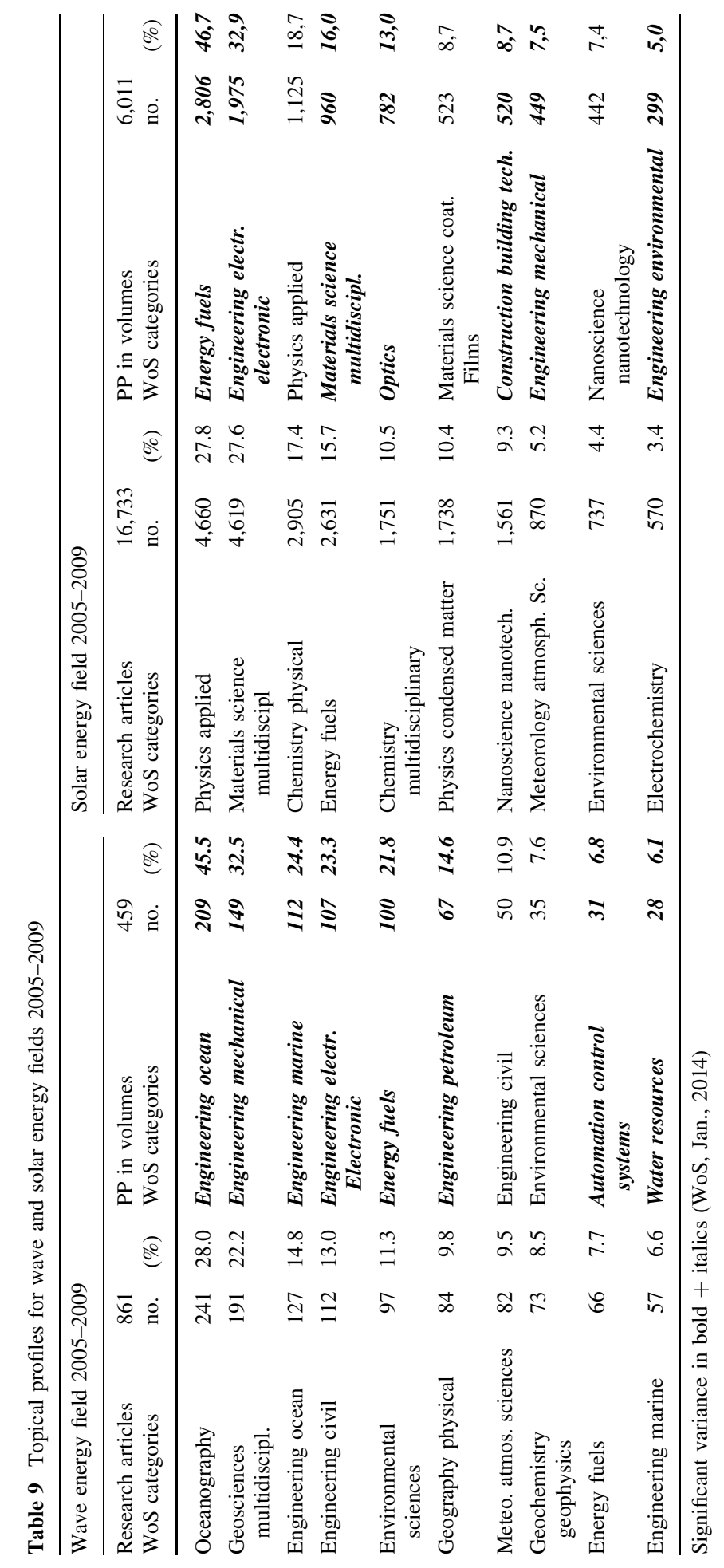


owing to a limited citation window of 3-4 years for that proportion but equally distributed across the fields.

The initial hypothesis that in strong proceedings paper dominant fields the proceeding papers themselves are the main contributor to the impact of the field or, at least, are the major supplier of citations to proceedings papers, does not hold entirely. Only the 'PP in journals' type in part behaves in accordance with our hypothesis: on the field impact, not as a major contributor of citations to the two proceedings paper types-and the 'PP in journals' type constitutes only on average $8.6 \%$ of the Renewable Energy publications.

The distribution of citations is highly asymmetric: All the document types investigated, including the two proceedings paper types, predominantly provide citations to the research articles-less to 'PP in journals' and almost none to the 'PP in volumes' type. Notwithstanding, in all analysed energy research areas positive associations are found in the observed values for "PP in volumes" as cited and citing documents and for "PP in journals" citing "PP in journals". Moreover, one observes the existence of a negative association in all disciplines of research articles citing "PP in volumes" and "PP in journals", for which the observed values are well below the expected ones by chance. These findings are independent of degree of proceedings paper dominance. 'PP in volumes' publications may consequently be regarded a significant (negative) player in the scientific communication process and thus a crucial factor in research evaluation, Fig. 1. With the exception of the proceedings paper dominant fields of Wind Power and Renewable Energy, and the Bio Mass field, in which the impact of 'PP in journals' surpasses that of research articles, the 'PP in journals' type contributes negatively in the four other disciplines to the overall field impact.

The following statistically significant trends are observed with decreasing proceedings paper dominance (and thus increasing journal article dependency) in the seven Renewable Energy fields:

(a) The probability increase that the field's overall citedness increases, Fig. 2 and Table 2;

(b) The ratio of 'PP in volumes' over 'PP in journals' decreases (from 13.7 in Wind Power to 1.6 in Bio Fuel, Table 1);

(c) The percentage of 'PP in volumes' citing 'PP in journals' over all publications citing 'PP in journals' decreases (from $26.3 \%$ in Wind Power to $4 \%$ in Bio Fuel, Fig. 2; Tables 3, 4, 5); and

(d) The segment of all proceedings papers (the combined 'PP' types) citing all proceedings papers over all publications citing all proceedings papers decreases (from $36.1 \%$ in Wind Power to $11.3 \%$ in Bio Fuel), Fig. 2, 3 and Tables 3, 4, and 5. This maximum share is close to the $40 \%$ found by Wainer de Oliveira and Anido (2011) in their reference analysis on the ACM Computer Science digital library.

These trends heavily contrast the initial hypotheses and speculations on proceedings paper citation provision in conference-dominant fields. It is noticeable that in the citedness game the country profiles may be influential. For instance, the Chinese focus on internationally scarcely cited 'PP in volumes' type in Wind Power, Tables 7 and 8, may indeed affect the overall impact of the field: A similar case is observed by He and Guan (2008) for proceedings papers in Chinese Computer Science. Probably, in fields with research article topical profiles concentrating on highly cited science-related categories and proceedings papers focussed on citation poorer engineering aspects the latter document type influences negatively on the field citedness as well as citation impact. 


\section{Conclusions}

Based on the findings it is recommendable not simply to rely on journal article analyses in comparative research assessment studies. All the research and innovation-producing types of documents should be taken into account in research evaluation. Such analyses should include proceedings papers-because this document type does have significant (negative or positive) influence on the overall citation impact of a research field, in particular in proceedings-dominant fields. In such fields the 'PP in journals' proceedings type may indeed positively support the overall impact score even though the 'PP in volumes' type commonly (in the Renewable Energy disciplines) influences negatively the outcome owing to a fundamental scarcity of citations. This recommendation may probably extend even to all engineering-like fields, but should be further investigated. At the same time the findings demonstrate that both types of proceedings papers and their impact pattern alone is not a good predictor of a highly or medium proceedings-dependent field's overall impact.

Acknowledgments This research was funded by the Spanish Ministry of Economy and Competitiveness under the project CSO2010-21759-C02-01 titled "Análisis de las capacidades científicas y tecnológicas de la eco-economìa en España a partir de indicadores cuantitativos y cualitativos de I + D + i” (Analysis of scientific and technological capacities of Eco-economy in Spain, throughout I + D + i quantitative and qualitative indicators), and by Carlos III University of Madrid-Banco de Santander Chairs of Excellence Program for 2011/2012 academic year. A shorter demonstration of some aspects of the analyses was presented in Ingwersen et al. 2013).

\section{Appendix A}

Annual distribution of published proceedings papers in the seven energy research fields (WoS, January 2014).

\begin{tabular}{|c|c|c|c|c|c|c|c|}
\hline $\begin{array}{l}\text { PP in } \\
\text { vol. }\end{array}$ & $\begin{array}{l}\text { Wind } \\
\text { power }\end{array}$ & $\begin{array}{l}\text { Renewable } \\
\text { energy }\end{array}$ & $\begin{array}{l}\text { Wave } \\
\text { energy }\end{array}$ & $\begin{array}{l}\text { Solar } \\
\text { energy }\end{array}$ & $\begin{array}{l}\text { Geo- } \\
\text { thermal }\end{array}$ & $\begin{array}{l}\text { Bio } \\
\text { mass }\end{array}$ & $\begin{array}{l}\text { Bio } \\
\text { fuel }\end{array}$ \\
\hline 2005 & 239 & 165 & 70 & 769 & 39 & 62 & 88 \\
\hline 2006 & 538 & 358 & 64 & 1,033 & 70 & 69 & 101 \\
\hline 2007 & 903 & 574 & 76 & 1,193 & 75 & 91 & 131 \\
\hline 2008 & 979 & 673 & 109 & 1,318 & 104 & 104 & 252 \\
\hline 2009 & 1,522 & 1,074 & 140 & 1,698 & 119 & 208 & 419 \\
\hline Total & 4,181 & 2,844 & 459 & 6,011 & 407 & 534 & 991 \\
\hline $\begin{array}{l}\text { PP in } \\
\text { jnls. }\end{array}$ & $\begin{array}{l}\text { Wind } \\
\text { power }\end{array}$ & $\begin{array}{l}\text { Renewable } \\
\text { energy }\end{array}$ & $\begin{array}{l}\text { Wave } \\
\text { energy }\end{array}$ & $\begin{array}{l}\text { Solar } \\
\text { energy }\end{array}$ & $\begin{array}{l}\text { Geo- } \\
\text { thermal }\end{array}$ & $\begin{array}{l}\text { Bio } \\
\text { mass }\end{array}$ & $\begin{array}{l}\text { Bio } \\
\text { fuel }\end{array}$ \\
\hline 2005 & 53 & 71 & 10 & 604 & 53 & 55 & 95 \\
\hline 2006 & 84 & 101 & 22 & 751 & 32 & 65 & 93 \\
\hline 2007 & 61 & 105 & 20 & 514 & 37 & 71 & 151 \\
\hline 2008 & 60 & 114 & 12 & 580 & 47 & 79 & 127 \\
\hline 2009 & 47 & 102 & 32 & 613 & 30 & 74 & 154 \\
\hline Total & 305 & 493 & 96 & 3,062 & 199 & 344 & 620 \\
\hline
\end{tabular}




\section{Appendix B}

Pearson's Residuals for cells in Tables 3, 4, and 5 in the seven energy research fields.

r_a: cited research article; re_a: cited review article; c_a: citing research article; c_pr_v: citing proc. papers in volumes; c_pr_j: citing proc. papers in journals; c_re: citing review articles

\begin{tabular}{|c|c|c|c|c|}
\hline & c_a & c_pr_v & c_pr_j & c_re \\
\hline \multicolumn{5}{|c|}{ Pearson's residuals: wind power } \\
\hline r_a & 1.4 & 1.9 & -3.0 & -5.5 \\
\hline PP_vol & -5.7 & 11.5 & -1.2 & -4.1 \\
\hline$P P_{-} j$ & -0.9 & 0.3 & 7.1 & -3.2 \\
\hline re_a & 0.9 & -10.2 & -0.6 & 17.4 \\
\hline \multicolumn{5}{|c|}{ Pearson's residuals: renewable energy research } \\
\hline r_a & 2.8 & -0.1 & -3.4 & -5.0 \\
\hline PP_vol & -8.1 & 23.7 & 1.0 & -3.8 \\
\hline$P P_{-} j$ & -4.3 & 5.4 & 10.7 & -0.8 \\
\hline re_a & 0.5 & -9.0 & -2.5 & 9.3 \\
\hline \multicolumn{5}{|c|}{ Pearson's residuals: wave energy research } \\
\hline r_a & 1.8 & -2.5 & -0.9 & -2.3 \\
\hline PP vol & -2.6 & 5.4 & 1.1 & 1.2 \\
\hline$P P_{-} j$ & -2.3 & 4.1 & 2.6 & 0.3 \\
\hline re a & -2.5 & 0.9 & -0.8 & 8.9 \\
\hline \multicolumn{5}{|c|}{ Pearson's residuals: solar energy } \\
\hline r_a & 5.7 & -2.5 & -8.7 & -10.0 \\
\hline PP_vol & -13.5 & 41.5 & 5.7 & -1.9 \\
\hline$P P \_j$ & -14.7 & 21.1 & 37.9 & -4.0 \\
\hline re_a & 3.3 & -21.1 & -13.9 & 23.4 \\
\hline \multicolumn{5}{|c|}{ Pearson's residuals: geo-thermal energy } \\
\hline r_a & 3.0 & -2.3 & -3.7 & -3.9 \\
\hline PP_vol & -1.2 & 6.4 & -0.2 & -1.0 \\
\hline$P P \_j$ & -2.4 & 1.3 & 9.0 & -0.7 \\
\hline re_a & -4.0 & 1.9 & 0.4 & 9.0 \\
\hline \multicolumn{5}{|c|}{ Pearson's residuals: bio-mass } \\
\hline r_a & 3.9 & -2.4 & -4.1 & -6.5 \\
\hline PP_vol & -2.9 & 16.6 & 2.1 & -2.6 \\
\hline$P P \_j$ & -3.2 & 3.6 & 9.5 & 1.0 \\
\hline re_a & -4.9 & 0.0 & 1.1 & 12.1 \\
\hline \multicolumn{5}{|c|}{ Pearson's residuals: bio-fuel } \\
\hline r_a & 4.0 & 0.9 & -1.2 & -10.8 \\
\hline PP_vol & -4.8 & 21.5 & 2.1 & -0.8 \\
\hline$P P_{-} j$ & -4.2 & 0.8 & 9.9 & 4.9 \\
\hline re_a & -2.7 & -4.2 & -4.3 & 12.5 \\
\hline
\end{tabular}




\section{References}

Bourke, P., \& Butler, L. (1996). Publication types, citations rates and evaluation. Scientometrics, 37(3), 473-494.

Butler, L., \& McAllister, I. (2009). Evaluating university research performance using metrics. European Political Science, 10, 44-58.

Butler, L., \& Visser, M. S. (2006). Extending citation analysis to non-source items. Scientometrics, 66(10), 327-343.

He, Y., \& Guan, J. C. (2008). Contribution of Chinese publications in computer science: A case study on LNCS. Scientometrics, 75(28), 519-534.

Ingwersen, P., \& Larsen, B. (2014). Influence of a performance indicator on Danish research production and citation impact 2000-12. Scientometrics, . doi:10.1007/s11192-014-1291-X.

Ingwersen, P., Larsen1, B., Garcia-Zorita, J. C., Serrano-López, A. E., \& Sanz-Casado, E. (2013). Contribution and influence of proceedings papers to citation impact in seven conference and journal-driven sub-fields of energy research 2005-11. In: Proceedings of the 14th Conference of the International Society of Scientometrics and Informetrics, Vienna, August 2013 (p. 418-425).

Martins, W. S., Goncalves, M. A., Laender, A. H. F., \& Ziviani, N. (2010). Assessing the quality of scientific conferences based on bibliographic citations. Scientometrics, 83(19), 133-155.

Meyer, D., Zeileis, A., \& Hornik, K. (2012). ved: visualizing categorical data. R package version 1.2-13.

Mutz, R., Bornmann, L., \& Daniel, H.-D. (2013). Types of research output profiles: a multilevel latent class analysis of the Austrian Science Fund's final project report data. Research Evaluation, 22(2), 118-133.

Sanz-Casado, E., Garcia-Zorita, J. C., Serrano-Lopez, A. E., Larsen, B., \& Ingwersen, P. (2013). Renewable energy research 1995-2009: a case study of wind power research in EU, Spain, Germany and Denmark. Scientometrics, 95(1), 197-224.

Thomson-Reuters. ISI. http://researchanalytics.thomsonreuters.com/essays/cpciessay/. Accessed 19 May 2014.

Wainer, J., de Oliveira, H. P., \& Anido, R. (2011). Patterns of bibliographic references in the ACM published papers. Information Processing and Management, 47(13), 135-142.

Waltman, L., Calero-Medina, C., Kosten, J., Noyons, E. C. M., Tijssen, R. J. W., van Eck, N. J., et al. (2012). The Leiden ranking 011/2012: Data collection, indicators, and interpretation. Journal of the American Society for Information Science and Technology, 63(12), 2419-2432. doi:10.1002/asi.22708. 